\title{
A Mitochondria-Cluster At The Proximal Axon Initial Segment Controls Axodendritic TAU Trafficking In Rodent Primary And Human iPSCderived Neurons
}

Noah Tjiang ( $\sim$ tjiang.n@gmail.com )

Universität zu Köln Medizinische Fakultät: Universitat zu Koln Medizinische Fakultat https://orcid.org/0000-0002-9957-0198

Hans Zempel

Universität zu Köln Medizinische Fakultät: Universitat zu Koln Medizinische Fakultat

\section{Research Article}

Keywords: axon initial segment/AIS, mitochondria, TAU, live-cell-imaging, Alzheimer's disease, neuron

Posted Date: July 12th, 2021

DOI: https://doi.org/10.21203/rs.3.rs-660779/v1

License: (c) This work is licensed under a Creative Commons Attribution 4.0 International License.

Read Full License 


\section{A mitochondria-cluster at the proximal axon initial segment controls axodendritic TAU trafficking in rodent primary and human iPSC- derived neurons}

Authors: Noah Tjiang ${ }^{1,2+}$ and Hans Zempel ${ }^{1,2 *}$

Noah Tjiang: https://orcid.org/0000-0002-9957-0198 ntjiang@ smail.uni-koeln.de Hans Zempel: https://orcid.org/0000-0002-7510-3077 hans.zempel@uk-koeln.de

Affiliations: ${ }^{1}$ Institute of Human Genetics, Faculty of Medicine and University Hospital Cologne, University of Cologne, 50931 Cologne, Germany

${ }^{2}$ Center for Molecular Medicine Cologne (CMMC), Faculty of Medicine and University Hospital Cologne, University of Cologne, 50931 Cologne, Germany

${ }^{+}$Submitting author and contact during editorial process

*Corresponding author

\section{Abstract}

Loss of neuronal polarity and missorting of the axonal microtubule-associated protein TAU are hallmarks of Alzheimer's disease (AD) and related tauopathies. Impairment of mitochondrial function is causative for various neurogenetic mitochondriopathies, but the role of mitochondria in tauopathies and in axonal TAU-sorting is still unclear. The axon initial segment (AIS) is vital for maintaining neuronal polarity and proper sorting of TAU.

Here, we aimed to investigate the role of mitochondria in the AIS regarding the maintenance of TAU polarity. Using global mitochondria impairment, but also live-cell-imaging and photoactivation methods, we specifically tracked and selectively impaired mitochondria in the AIS in primary mouse and human iPSC-derived neurons, and measured the subsequent missorting of TAU.

We observed that global application of mitochondrial toxins efficiently induced tauopathy-like missorting, indicating involvement of mitochondria in TAU polarity. Mitochondria show a biased distribution within the AIS, with a proximal cluster and relative absence in the central AIS. The mitochondria of this cluster are largely immobile and only sparsely participate in axonal mitochondria-trafficking. Locally constricted impairment of only the AIS-mitochondria-cluster leads to detectable increases of somatic TAU, reminiscent of AD-like TAUmissorting.

Here, we provide first evidence that the mitochondrial distribution within the proximal axon is biased towards the proximal AIS and that proper function of this newly described mitochondrial cluster may be essential for the maintenance of TAU neuronal polarity. This strengthens the role of mitochondrial impairment as an upstream event and therapeutic target in the pathological cascade leading to TAU missorting and consequent neuronal dysfunction.

\section{Keywords}

axon initial segment/AIS; mitochondria; TAU; live-cell-imaging; Alzheimer's disease; neuron

\section{Declarations}

Funding: Else-Kroener-Fresenius-Stiftung, Koeln Fortune.

Conflict of interest/competing interests: The authors declare no conflicts of interest and no competing interests.

Data availability: The original contributions presented in the study are included in the article/supplementary material, further inquiries can be directed to the corresponding author.

Code availability: Not applicable.

Ethics approval: Primary mouse neuron culture generation was reviewed and approved by Carolin Debuschewitz, Animal Welfare Officer of the University of Cologne (according to §4 Tierschutzgesetz).

\footnotetext{
Abbreviations

AD Alzheimer's Disease, AIS axon initial segment, AP action potential, hiPSC human-induced-pluripotent stem cells, MPD Mito-Photo-DNP, TBD TAU-diffusion-barrier, TTLL6 Tubulin-Tyrosine-Ligase-Like-6.
}

\section{Acknowledgments}

See page 8 . 


\section{Introduction}

Alzheimer's disease (AD) and other tauopathies are neurodegenerative disorders that impose a huge burden on the aging society [1]. Pathological hallmarks of these tauopathies are abnormal phosphorylation, subcellular mislocalization and eventually, aggregate formation (mainly neurofibrillary tangles) of the microtubule-associated protein TAU [2]. Understanding the detrimental cascade of these TAU alterations is crucial for developing effective therapies and prevention options. However, the underlying mechanisms remain largely elusive.

In healthy neurons, TAU is sorted into the axonal compartment but in disease conditions, this axonal targeting of TAU fails, and the subsequent somatodendritic accumulation of TAU results in various pathological effects, ranging from protein-aggregation-based sequestering of various proteins to the mislocalization and aberrant activation of other proteins like FYN or TTLL6. Furthermore, TAU missorting leads to the loss of microtubules and mitochondria, all of which leads to neuronal damage, neuronal dysfunction and ultimately neurodegeneration [2]. While the mechanisms of axonal TAU sorting are still under debate $[3,4]$, there is evidence for a major role of the axon initial segment (AIS) for mediation of axonal TAU targeting [5,6]. The AIS is a 20-60 $\mu \mathrm{m}$ long segment at the proximal axon, and the site of action potential (AP) generation [7]. The AIS is characterized by a highly structured cytoskeleton with enrichment of specific proteins (e.g. ANKRYIN-G, TRIM-46) and voltage gated potassium/sodium channels [8].

Multiple studies have shown that the knockdown of AIS components (e.g. ANKYRIN-G) results in mistrafficking of TAU, implicating the relevance of an intact AIS for the maintenance of neuronal health and polarity [5,9]. Studies with rodent primary neurons demonstrated that the AIS composes a TAU-diffusion barrier (TBD) $[5,10]$ that controls retro- and anterograde trafficking of TAU. The mechanisms behind this TBD are largely enigmatic.

Besides losing proper axonal sorting of TAU, tauopathies and models thereof are associated with defects in mitochondrial function [11,12]. Normally, mitochondria fulfil crucial tasks such as the homeostasis of calcium, of reactive oxygen species (ROS) and the generation of ATP. They are steadily recycled via mitophagy and biogenesis, which mainly happens in the soma of neurons [13]. The disease cascades of tauopathies lead to alterations of the mitochondrial homeostasis, e.g. an increase of fission proteins such as dynamin-related protein 1 (DRP1) [14] which lead to excessive production of ROS and altered mitochondrial trafficking [15]. This disrupts the (re-)distribution of mitochondria into the meter-long neurites which in turn intensifies the decline of neuronal health in these diseases $[16,17]$.

When modelling the contributions of mitochondria as disease meditators, the global mitochondrial impairment through external factors or genetic mutations alone is sufficient to achieve tauopathy-like neurodegeneration like TAU-hyperphosphorylation and TAU-missorting both in vitro and in vivo [18]. The local effects of mitochondria and their potential dysfunction, especially in areas that are crucial for efficient TAU sorting - like the AIS however, are unstudied.

In this study, we describe a mitochondrial cluster in the proximal AIS present in murine primary cortical neurons as well as in human iPSC-derived cortical neurons, which influences axonal TAU sorting. The mitochondria of this AIS-cluster participate in fewer trafficking events and are less mobile compared to other axonal mitochondria. By inducing mitochondrial impairment specifically in this cluster, we could demonstrate that efficient TAU sorting relies on the proper function of these specific mitochondria. Our results suggest a key role of AIS-located mitochondria for the maintenance of TAU polarity and give insights into disease mechanism of AD and other tauopathies. 


\title{
Materials and Methods
}

\author{
Molecular biology
}

To obtain a photoconvertible mitochondrial marker, we replaced the fluorescent protein sequence of mito-RFP with a sequence encoding Dendra2c via restriction cloning using SalI and BgIII. To avoid spectral overlap in the live imaging experiments, a far-red fluorescent edition of the AIS-marker YFP-Nav-II-III (a gift from Matthew Grubb; Addgene plasmid \#26056) was created by cloning the NaV-sequence into the vector of emiRFP670 (a gift from Vladislav Verkhusha; Addgene plasmid \#136556) using NotI, SacI and mutagenesis-PCR.

\section{Cell culture and Immunostaining}

All experiments involving animals were conducted according to local legislation and animal welfare guidelines. Mouse primary neurons (MPNs) were prepared as described and characterized [5,19]. Briefly, forebrain neurons were isolated by dissecting embryonic day $13.5 \mathrm{FVB} / \mathrm{N}$ mice and isolating the cortical hemispheres after extraction of the brain. These were digested with 1x Trypsin (Panbiotech) and diluted in neuronal plating medium (Neurobasal medium, 1\% FBS, 1x Antibiotic/Antimycotic Solution; ThermoFisherScientific (TFS), 1x NS21 (Panbiotech)), plated in in a either 24-well-plate (VWR) onto coverslips pre-treated with poly-dlysine (PDL; Sigma-Aldrich) or a PDL-treated "IBIDI- $\mu$-Dish $35 \mathrm{~mm}$, high Grid-500 Glass Bottom" for live imaging/re-locating cells and then kept in a humidified incubator at $37{ }^{\circ} \mathrm{C}$ and $5 \% \mathrm{CO}_{2}$. After four days, the same amount of neuronal maintenance medium (Neurobasal media (TFS), 1× Antibiotic-/Antimycotic solution (TFS), $1 \times$ NS21 (Panbiotech) and AraC (Sigma-Aldrich)) was added and cells were kept in culture as long as indicated. Human WTC11 iPSCs carrying a doxycycline-inducible Neurogenin2 (Ngn2) transgene were differentiated into neurons as previously described and characterized [20-22].

MPNs and iPSC-derived neurons were transfected according to the manufacturers protocol with PolyJet (tebubio/Signagen) and Lipofectamine Stem Transfection Reagent (TFS), respectively, and plasmids were expressed for the indicated time period.

Cells were fixed and stained as previously described [19]. In brief, they were fixed with $3.7 \%$ formaldehyde (Sigma-Aldrich; in PBS), blocking and permeabilization were achieved by incubating for 5 minutes with $0.5 \%$ Triton-X and $10 \%$ bovine serum albumin in PBS. Primary antibodies were incubated overnight at $4{ }^{\circ} \mathrm{C}$, secondary antibodies at RT for $1 \mathrm{~h}$. After adding NucBlue (TFS) to stain nuclei, coverslips were mounted using AquaPolyMount (Polysciences). The following antibodies were used:

\section{Primary antibodies}

mouse-anti-ANKYRIN-G

rabbit-anti-TRIM-46

chicken-anti-MAP2

rabbit-anti-TAU

mouse-anti-MTCO1

\section{Secondary antibodies}

donkey-anti mouse 647

donkey-anti mouse-561

donkey anti-chicken 647

donkey anti rabbit 561

donkey-anti-Chicken CF488A IgY (H+L)

\author{
Neuromab/Biozol N106/36, \#75-146 \\ Synaptic Systems, \#37703 \\ Abcam, \#ab5392 \\ Dako, \#A0024 \\ Invitrogen, 1D6E1A8, \#459600
}

AlexaFluor (Invitrogen)

AlexaFluor (Invitrogen)

AlexaFluor (Invitrogen)

AlexaFluor (Invitrogen)

Linaris, \#20166

$\begin{array}{ll}\text { monoclonal } & 1: 250 \\ \text { polyclonal } & 1: 500 \\ \text { polyclonal } & 1: 1000 \\ \text { polyclonal } & 1: 1000 \\ \text { monoclonal } & 1: 500 \\ & \\ & 1: 1000 \\ & 1: 1000 \\ & 1: 1000 \\ & 1: 1000 \\ & 1: 1000\end{array}$

Cells were treated as indicated with Rotenone or Antimycin A dissolved in Ethanol, diluted with cell culture medium; control cells were treated with an equal amount of carrier solution. Somatodendritic TAU fluorescence intensity was measured in a semi-automatic way by detecting somatodendritic regions of interest (ROI's) via MAP2, the mean background fluorescence intensity of the image was subtracted, and treated cells were normalised to control cells.

\section{Imaging and photoactivation/conversion}

Epifluorescence microscopy was performed with a Zeiss axioscope equipped with Colibri 7 LED and an Axiocam 503 mono. Preparation for STED-nanoscopy followed the recommendations from the manufacturer, imaging was 
conducted with a Leica gSTED TCS SP8 system using AntifadeGold mounting medium (Invitrogen). Confocal fluorescence, live-cell-imaging and photoconversion/photoactivation (see below) was done using a Perkin ElmerYokogawa CSU-X1-Spinning-Disk-Confocal-Microscope.

After identifying the AIS and the corresponding mitochondria (using the NaV-emiRFP-670), mito-Dendra in the selected ROI was converted with the $405 \mathrm{~nm}$-laser of the integrated FRAP tool. Images were acquired in both green and red channels every $2-5$ seconds as a 3 -layered z-stack (step size $1 \mu \mathrm{m}$ ) for 30 minutes. Non suitable cells (e.g. no cotransfection or no mitochondrial movement) were excluded from analysis.

To find out the appropriate parameters for the successful photo-activation of mito-photo-DNP (MPD) in neuronal cells, we added the live-imaging mitochondrial-membrane-potential $(\triangle \Psi)$ marker tetramethyl-rhodamine-methylester (TMRM; 10nM; TFS) to the culture and titrated the adequate laser powers and concentration of MPD where TMRM fluorescence and thus $\Delta \Psi$ would significantly drop, indicating mitochondrial disruption. Subsequent experiments were carried out without TMRM using the established parameters.

Cells were transfected with mito-RFP and NaV-emiRFP-670 to identify the AIS and corresponding cluster, after selecting adequate cells and ROI's, 200nM of MPD (BioTrend, dissolved in DMSO (Carl Roth)) was added and after 20 min of incubation, photo-activation in the ROI was achieved with a 355nm-Laser (Rapp-OptoElectronic). Cells were kept in the incubator after treatment for $1.5 \mathrm{~h}$ before fixing and staining for TAU. For control cells, MPD was either activated in the soma with a ROI of comparable size or the laser was used on the AISmitochondria-cluster before the addition of MPD. TAU fluorescence intensity was then measured in the soma of treated cells and normalized to that of untreated cells $(\mathrm{Ctrl})$ in the same dish.

\section{Data Analysis and Statistics}

Image analysis was done using Fiji-ImageJ [23], graphing and statistical analysis was performed using GraphPadPrism 8. Data were analysed for normal distribution and statistical tests were performed accordingly (for details on statistical tests, see corresponding figure legend).

Cluster prevalence assessment was done by categorizing each valid cell using three criteria: 1 . mitochondria present in the proximal AIS (range $\pm 7.5 \mu \mathrm{m}$ ); 2. relative absence of mitochondria in the central AIS; 3 . visible accumulation/higher brightness of the mitochondria in the proximal AIS. Cells were then categorized into 3 groups: cluster (+): 3 criteria met; cluster ( ): 2 criteria met; and cluster $(-): \leq 1$ criterion. The ratio was calculated as Ratio $=\frac{n_{\text {Total }}}{\left(n_{(+)}+0,5 * n_{(\sim)}\right)}$.

The mitochondrial distribution along the AIS was measured by drawing in a line from the soma through the axon and creating a plot profile of the intensity of ANKYRIN-G. This was smoothened using a $1 \mu \mathrm{m}$ sliding-mean and the "starting point" of the AIS was set to the point where this sliding mean was first $\geq 30 \%$ of its maximum (adapted from Dumitrescu et. al. 2016) [24].

To account for the difference in fluorescence intensity between cells and the high background fluorescence near the soma, mito-RFP images were thresholded using Trainable Weka segmentation (Arganda-Carreras, 2017), plot profiles from these binary pictures were generated with the same line-ROI's, fitted to the "starting point" of the AIS and then averaged over all cells. Because the ROI's had differing lengths, $n$ got lower towards the extremes, so this data was not used. To statistically test the difference in distribution, the mean of each replicate over a span of $10 \mu \mathrm{m}$ was used and the distance between the two lines was kept the same in Fig. $2 \mathrm{~h}$ and $2 \mathrm{i}$.

While segmenting the images into binary pictures solved the problem of varying fluorescence intensity, this eliminated a property of the AIS-mitochondria cluster: most often it was brighter than comparable mitochondria in the more distal AIS, a feature that is not reflected by this quantification.

Kymographs were created using the KymographBuilder plugin in Fiji-ImageJ. All trafficking events that went past the cluster were counted, categorized into red/green and antero-/retrograde and expressed as a fraction of each cells total trafficking events. 
Results

\section{Global mitochondrial impairment leads to deficient TAU-sorting}

Chronic exposure of neurons to mitochondrial toxins leads to TAU hyperphosphorylation and aggregation $[12,18]$. The acute effect of mitochondrial impairment on TAU sorting, however, is unclear. We aimed to investigate TAU intracellular localization after mitochondrial impairment by using mitochondrial stressors. First, we treated mouse primary neurons (MPN; 21 days in vitro (DIV)) with the respiratory chain inhibitors Rotenone (ROT) and Antimycin A (AMA) for 2 hours. We quantified the levels of endogenous TAU in the soma using MAP2 (microtubule associated protein 2) as somatodendritic marker (Fig. 1a-c). We observed a substantial difference in somatodendritic TAU levels after exposure to the mitochondrial toxins, indicating a failure of effective axonal TAU sorting. This was most striking after treatment with $50 \mathrm{nM}$ AMA (approx. 18-fold higher TAU signals in cells somata, 13-fold for Rotenone; Fig. 1d). Longer treatment duration and higher doses resulted in elevated levels of cytotoxicity and cell death (data not shown). Altogether, these results show that an acute, global inhibition of mitochondrial function can induce profound somatodendritic TAU missorting, outclassing previously reported AD-like missorting induced by Amyloid- $\beta$ by a factor of 5-10 (e.g. Zempel et al. 2013) [25].

\section{A mitochondrial cluster is localized at the proximal axon initial segment}

As both the AIS [5][6] and mitochondrial function are crucial for proper TAU sorting (Fig. 1), we examined mitochondrial localization and dynamics in the AIS. First, we transfected both MPN's (Fig. 2a) and hiPSCderived-neurons (Fig. 2b) with mito-RFP, immunostained for the AIS-proteins ANKYRIN-G/TRIM46 to outline the AIS-position and measured the mitochondrial distribution along the AIS. This was done at four different timepoints after the initial establishment of neuronal cell polarity (meaning past "Banker stage" IV) [26].

In approx. $40 \%$ of the analysed neurons we observed a characteristic pattern of mitochondrial distribution in the AIS. It consists of a cluster-like aggregation of mitochondria in the proximal AIS (arrowheads, Fig. 2a-b) and a subsequent zone in the central AIS that showed a lower abundance of mitochondria (arrows, Fig. 2a-b). This "AISmitochondria-cluster" coincided with the beginning of the AIS within a range of $7.5 \mu \mathrm{m}$. We found this distinct mitochondrial distribution in both MPN and in hiPSC-derived neurons (Fig. 2a-b).

We further validated our initial observation of an AIS-specific mitochondrial cluster using two-colour STED super resolution microscopy. We used mito-YFP and the uniformly distributed volume marker mTFP in superresolution mode (Fig. 2, c1-c3). We confirmed the mitochondrial clustering in the proximal AIS and observed that the AISmitochondria-cluster is relatively large, leaving only a space of 70-300 nm to the sides. The mitochondria-cluster is not composed of a single, large mitochondrion, but rather several clearly distinguishable mitochondria (Fig 2d$\mathrm{f}$; for a schematic depiction of the mitochondrial distribution within the AIS see Fig. $2 \mathrm{~g}$ ).

We quantified and averaged the mitochondrial distribution along the AIS in MPNs and determined that the local mitochondrial prevalence was up to 10 times higher in the proximal part of the AIS than in the central part. Even when averaged over a span of $10 \mu \mathrm{m}$, the prevalence was still about a 2.5-fold higher (Fig. 2h). Similar measurements along dendrites showed no significant differences in mitochondrial distribution (Fig. 2i), demonstrating that this is a property unique to the AIS.

Next, we examined the occurrence of this AIS-mitochondria at different time points of the neuronal maturation. For this, we transfected and fixed MPN at different days in-vitro (Fig. 2j). The mitochondrial cluster was observable throughout our examined time course, with $\sim 33 \%$ of neurons expressing it already after 7 DIVs and an increase in frequency to $43 \%$ in older neurons, although these results did not reach statistical significance.

\section{Mitochondria within the AIS-mitochondria-cluster are immobile}

As mitochondria show net anterograde trafficking into the axon and the mitochondria-cluster is composed of several mitochondria, we hypothesized that it serves as a spawning hub for generating axonally trafficked mitochondria. We hence examined the dynamics of the AIS-mitochondria-cluster by co-transfecting mito-Dendra (a photoconvertible fluorescent protein that we fused to a mitochondrial targeting sequence) and the AIS marker NaV-emiRFP670 [24] into MPN (DIV7), and assessed mitochondrial mobility after four days of expression. We identified the AIS (using NaV-emiRFP670) and the corresponding cluster (using mito-Dendra) in live-cellmicroscopy, photoconverted the mitochondria within the AIS-mitochondria-cluster from green to red, and conducted time-lapse-imaging (Fig. 3a-b). 
This approach allowed us to distinguish between AIS-located mitochondria (red Dendra2) and mitochondria originating from other cell compartments (green Dendra2). Interestingly, we did not observe a single event when the whole AIS-mitochondria-cluster moved or dissipated in all our imaging sessions. Moreover, we observed axonal transport of mitochondria from the soma that passed through the proximal cluster at multiple times, seemingly without affecting it at all (Fig. 3c, cyan lines). In total, trafficking that originated from the AISmitochondria-cluster only made up $\sim 15 \%$ of all trafficking events in the AIS, while the other $85 \%$ originated from mitochondria from the axon and/or soma (Fig. 3d). In line with previous findings [27], we also saw that anterograde trafficking of mitochondria was more frequent than retrograde (here: about $40 \%$ more). Retrograde trafficking almost exclusively ( 98\%) originated from mitochondria further down the axon and not from the AISmitochondria-cluster.

Overall, this indicates that the cluster of mitochondria is anchored at the proximal AIS and does not serve as a hub for the axonal trafficking of mitochondria.

\section{The AIS-mitochondria-cluster plays a functional role in the maintenance of axonal TAU sorting}

Since i) the mitochondrial cluster is localized right at the AIS - a region crucial for maintaining neuronal polarity and proper sorting of TAU [5,6], and ii) cell-wide inhibition of mitochondria leads to impairment of these processes (namely TAU-missorting in our model, see above), we next tested whether the cluster of mitochondria might be involved in maintaining TAU cellular polarity.

We used the compound Mito-Photo-DNP (MPD) that allows precise, spatially and timely defined disruption of the mitochondrial membrane potential upon photoactivation in living cells, as previously reported [28]. To test our setup, we incubated MPN with 200nM MPD and 10nM TMRM, a live-imaging mitochondrial-membrane-potential marker. After photoactivation, mitochondrial membrane potential was strongly and stably reduced at the illuminated ROI (ROI A), while remaining unaffected in regions of the cell without photoactivation (ROI B, Fig. $4 a-b)$.

Next, we tested the effect of local AIS-mitochondria-cluster impairment on sorting of TAU. We used DIV18 hiPSC-derived neurons and DIV11 MPNs (both of which show mature neuronal polarity and effective TAU sorting into the axon at this age) cotransfected with mitoRFP and NaV-emiRFP670 for 4 days ( 7 days for hiPSC-derived neurons) to detect the cluster within the neurons (Fig. 4c-d). We disrupted regional mitochondrial function by photoconversion of MPD at the AIS-mitochondria-cluster (Fig. 4c-d). After 1.5 hours, cells were fixed and TAU levels in the soma were measured and compared to non-treated control cells via immunostaining (Fig. 4e).

A small but significant increase in somatodendritic TAU-levels was observed after impairment of AIS-localized mitochondria (Fig. 4f-g) compared to untreated cells ( 15\% increase in MPNs and $\sim 12 \%$ in iPSC-derived neurons). This effect is specific to axonal/AIS mitochondria since interference with somatic or dendritic mitochondria did not result in TAU missorting (Suppl. 2). Altogether, this indicates a crucial role of the small subpopulation of mitochondria anchored in the proximal AIS for the maintenance of axonal sorting of TAU and may establish them as a vulnerable player in the complex disease cascades of tauopathies.

\section{Discussion}

In this study, we investigated the relation of mitochondrial (dys-)function and TAU (mis-)sorting. We focussed on mitochondria within the AIS, as this is the currently suggested crucial site for proper TAU sorting and maintenance of neuronal polarity.

We described a characteristic pattern of mitochondrial distribution along the AIS, which consists of a proximal cluster of mitochondria and a region in the central AIS that is mostly devoid of mitochondria. The mitochondria of the cluster also frequently appear to be brighter than the average mitochondria along the axon, hinting towards a larger mitochondrial mass and number at that site. This is in line with our observations from STED-nanoscopy, which showed several densely packed mitochondria at the proximal AIS. We observed this cluster in both primary rodent forebrain as well as human iPSC-derived cortical neurons, indicating that this is a conserved feature across species and may be an important characteristic of the AIS - at least in cortical neurons. Interestingly, this "phenotype" of cells exhibiting the AIS-mitochondria-cluster (as defined by the selected criteria, see Methods) was not observed in all the cells examined, but rather in about 30-45\%. This fraction is roughly consistent over the development of the neurons, indicating that the AIS-mitochondria-cluster may be rather independent of neuronal maturation after the establishment of the AIS. Defined subpopulations of neurons that express this characteristic pattern of mitochondria seem plausible given that rodent forebrain cultures are still a heterogeneous mix of neurons when compared to our hiPSC-derived neurons [20]. On the other hand, our preliminary observations suggest that the AIS-mitochondria-cluster is not observable in all hiPSC-derived neurons either, even though they are very homogenous [20]. This indicates that the mitochondria-cluster is clearly observable only in a subset of primary 
but also homogenous clonal cells, thus the differences might stem from variable physiological characteristics of the AIS. These depend on the individual electrophysiological properties, differential network integration and varying places of origin of the AIS, but are also distinct in different subpopulation of neurons [29].

Mitochondria tend to accumulate in regions of high energy demand, so the mitochondria may be clustered in the AIS to support the local generation of action potentials (AP). One reason to why they are not evenly distributed along the AIS might be the varying distribution of different Nav channels: Nav1.6 channels (responsible for AP generation) and high-threshold Nav1.2 channels are also enriched in two distinct groups along the AIS [30]. Future studies may investigate whether the AIS-mitochondria-cluster colocalizes with a specific subgroup of Nav channels and whether they functionally interact.

We observed that the mitochondrial cluster is stationary at the proximal AIS in live-cell-imaging. The results from these experiments confirm the prior observation made in fixed cells and rule out that these observations were artifacts created by a zone of slower mitochondrial transport, as mitochondria still moved through the AIS while the cluster remained stationary, even when we tentatively increased imaging time up to several hours. In line with previous studies [31], exploratory experiments showed that photoconversion in itself did not cause reduced mitochondrial motility, ensuring that we did not create unwanted artifacts.

Our initial hypothesis - that the AIS-mitochondria-cluster might be a spawning hub for generating axonally trafficked mitochondria - was refuted since the AIS-mitochondria-cluster only sparsely participates in mitochondrial trafficking. There, the mismatch between the small number of photoconverted mitochondria and relatively large number of non-photoconverted mitochondria could have falsely amplified our result, but given the small scale of both the observed area and relatively short timeframe, this bias should be small and not be able to cause the observed large difference in trafficking. Nevertheless, it remains puzzling how mitochondria from the soma and/or axon are passing the proximal cluster without affecting it, even though the space around the cluster appears to be very narrow $(<300 \mathrm{~nm}$ in STED-nanoscopy). A possible explanation would be that trafficking mitochondria follow the microtubule tracks in the centre of the axon, while the AIS-mitochondria-cluster could have a ring-like structure in the cross-section, allowing mitochondria to pass both ways. A former study using electron microscopy supports this hypothesis, showing that the mitochondria that "accumulate in the proximal segment of the axon [...] occupy the peripheral regions of the axoplasm" [32], although this study was limited to rat hypoglossal nerve.

Subsequently, it will be important to understand the cytoskeletal scaffold that might anchor the AIS-mitochondriacluster in place. The overall mobility of membrane proteins is greatly reduced in the AIS, mainly because of the intertwined ankyrin-spectrin-actin-cytoskeleton [33,34]. The AIS-mitochondria-cluster might interact with one of these AIS-specific scaffold proteins (e.g. actin via myosin) [35] or with syntaphilin-like proteins, which were found as a docking partner of axonal mitochondria in rat hippocampal neurons [36]. In-vivo-proximity-labelling of local interaction partners of the AIS-mitochondria-cluster could be a valuable experiment to investigate anchoring mechanisms.

Studying the functional aspects of the mitochondria-cluster in the proximal AIS, we found that local impairment of the AIS-mitochondria-cluster alone is sufficient to induce subtle, but detectable increase of somatic TAU protein. This TAU-missorting after local mitochondrial impairment was not as striking as the effect of global mitochondrial inhibition through ROT/AMA (see also Suppl. 1), but this difference was expected since the AISmitochondria-cluster makes up less than $0.1 \%$ of the cell's total mitochondria (as estimated by mito-RFP-positive area).

In fact, these results suggest that the observed TAU-missorting after local impairment is an effect specific to the cluster-mitochondria, since they make up less than $0.1 \%$ of the cells mitochondria but lead to more than $1 \%$ of the missorting observed after global inhibition. This hypothesis is further supported by the fact that we did not observe measurable TAU-missorting when impairing a comparable small number of mitochondria in the somatodendritic compartment (Suppl. 2). Therefore, it is probable that the observed TAU-missorting after local impairment of AISlocalized mitochondria is due to a disruption of local processes in the AIS.

The mechanisms responsible for the observed TAU-missorting may be i) impaired anterograde sorting of TAU at the AIS, or ii) disruption of the retrograde barrier that holds axonal TAU in place. On the anterograde side, the locally impaired mitochondrial homeostasis could impair the microtubule-based, anterograde transport of TAUmRNA [37] and also the energy demanding, local TAU-protein-translation, proposed to mainly take place in the AIS [5].

On the other side, the locally reduced level of readily available energy providers (i.e. nucleoside triphosphates such as ATP) could also alter the functions of glycogen synthase-kinase-3 $\beta$ (GSK3 $\beta$ ). GSK3 $\beta$ is a major kinase tethered in the AIS and is implicated with disease pathology in AD and dysfunction of the TBD [38,39]. This 
theory is further supported by the finding that GSK3 $\beta$ is also activated by ROS [40]. It is very plausible that the local level of ROS is higher after our impairment of regional mitochondria, which would result in two different ways of how dysfunction of the AIS-mitochondria-cluster can lead to failure of the TAU diffusion barrier via alteration of GSK3ß. These two different functions of the AIS-mitochondria-cluster (energy and ROS homeostasis) have to be further investigated in follow-up studies.

All in all, we provide evidence for a cluster of mitochondria in the proximal AIS in different types of cultured neurons that is largely stationary and has a functional role in the maintenance of effective TAU sorting. These location-specific mitochondria may play a crucial role in the pathomechanisms of tauopathies such as Alzheimer's disease and even in genetic mitochondriopathies. Thus, mitochondrial dysfunction may be upstream of TAU pathology in Alzheimer's disease and related tauopathies, and may be a relevant therapeutic target in the future.

\section{Acknowledgements}

We are grateful towards Michael Bell and Sarah Bachmann for their methodical advice and critical manuscript correction. We thank Jennifer Klimek for excellent technical assistance, Helen Breuer for a technical replicate, Julia Oly and Bianca Helling for initial experiments with mitochondrial toxins, Prof. Dr. R. Wiesner for providing TMRM and Brian Glancy, Ph.D. for his advice on using Mito-PhotoDNP. Live-cell microscopy was done at the CECAD Imaging Facility (Cologne, Germany).

Supported by the Koeln Fortune Program, Faculty of Medicine, University of Cologne and the Else-KroenerFresenius-Stiftung.

The left scheme in Fig. $2 \mathrm{~g}$ is adapted from an image by user Looxix available under the GNU Free Documentation License, version 1.2.

\section{Figure Legends}

\section{Fig. 1 Global mitochondrial impairment leads to TAU missorting}

a-c: Immunofluorescence staining of murine primary neurons (DIV21) for MAP2 (somatodendritic marker) and TAU, treated for 2 hours with (a) control, (b) 50nM Antimycin (AMA) or (c) 50nM Rotenone (ROT, both respiratory chain inhibitors), representative epifluorescence images.

Arrows in (a) indicate low level of somatic TAU in control cells, inserts show magnifications of exemplary cell bodies; arrowheads in (b-c) indicate missorted TAU in the soma after treatment, soma shows higher TAUfluorescence-intensity, therefore merge appears yellowish to green.

d: Quantification of TAU-fluorescence-intensity in the soma after subtraction of background fluorescence, arithmetic mean of 3 biological replicates with standard deviation (SD). Control: Mean=1.0; n=122-126; AMA: Mean=17.51 n=42-172; ROT: Mean=12.87; n=144-154. Ordinary one-way ANOVA with Dunnett's correction for multiple comparisons, Ctrl vs. AMA: **, $\mathrm{p}=0.0059$, Ctrl vs. ROT: * $\mathrm{p}=0.0258$, significance level $\mathrm{p}<0.05$.

\section{Fig. 2 Mitochondria cluster at the proximal AIS in cortical rodent and human neurons}

a-b: Representative images of DIV11 MPN (a) and DIV18 hiPSC-derived neurons (b) that were transfected with mito-RFP and immunostained for MAP2 (somatodendritic marker, magenta) and ANKYRIN-G/TRIM-46 (AISmarker, cyan): Asterisks indicate the cell body, dashed lines indicate the AIS where MAP2 enrichment stops and AIS-markers are present; mitochondrial distribution magnified in lower row: arrowhead shows proximal cluster of mitochondria in the AIS, arrow indicates the region of lower mitochondrial abundance in the central AIS. c-f: STED nanoscopy pictures of rat primary cortical neurons. Neurons aged 8-9DIV were transfected with mitoYFP (green) and mTFP (as a volume marker, red) for $2 \mathrm{~d}$, fixed and processed for two-colour STED nanoscopy.

Arrow in (c) indicates beginning of the AIS (by decreasing intensity of MAP2, c1), dashed lines indicate magnifications in inserts in d-f.

d-f: Magnification of the axon, arrowhead shows proximal cluster of mitochondria, arrow shows subsequent region of lower mitochondrial abundance in the central AIS. Inserts show second magnification of the proximal AIS. Measurement of the shortest distance between the mitochondria and cell border (by mTFP) shown on either side shown in "Magnific."

g: Scheme of a neuron, red box shows enlarged section of the proximal axon shown on the right: schematic of the observed mitochondrial distribution along the proximal axon, colouring indicates levels of MAP2 (magenta) and ANKYRIN-G (cyan) enrichment at the transition from soma to axon, cluster of mitochondria in the proximal AIS followed by a region of low mitochondrial abundance in the central AIS (marked by arrow).

h: Quantification of mitochondrial distribution along the AIS of MPN transfected with mito-RFP on DIV8, fixed 
after 3d, and immunostained for ANKRYIN-G. Probability of a presence of mitochondria as a fraction, biol. replicate weighted mean (blue), standard deviation (green) and $\sim 1 \mu \mathrm{m}$ walking average (black). A region of higher mitochondrial abundance (cluster, proximal AIS) is clearly distinguishable from a region of low mitochondrial presence (central AIS). Mean ${ }_{\text {Cluster }}=0.53$; Mean ${ }_{\text {Centre}}=0.22$; unpaired t-test with Welch's correction: **, $\mathrm{p}=0.0048,3$ biological replicates with $\mathrm{n}=11-16$, (for details see Methods).

i: Similar procedure to (h) but measured over randomly selected dendrites as a control. Probability of a presence of mitochondria as a fraction, biol. replicate weighted mean (blue), standard deviation (green) and $\sim 1 \mu \mathrm{m}$ walking average (black). Mean $_{\text {Start }}=0.50 ;$ Mean $_{\text {Centre }}=0.42$; unpaired t-test with Welch's correction: not significant, $\mathrm{p}=0.1278,3$ biological replicates with $\mathrm{n}=17-20$.

j: Ratio of cells showing the cluster morphology at different points in development, MPN were transfected with mito-RFP, fixed, stained for ANKYRIN-G, and classified according the appearance of the cluster (more details see Methods). Mean with SD, ordinary one-way ANOVA with Tukey's correction for multiple comparisons: not significant, 3 biological replicates each, $\mathrm{n}$ for each replicate: $\mathrm{n}_{\operatorname{div} 7}>21, \mathrm{n}_{\operatorname{div} 11}>18, \mathrm{n}_{\operatorname{div} 14}>15, \mathrm{n}_{\operatorname{div} 21}>5$.

\section{Fig. 3 The mitochondrial AIS-cluster is immobile and only sparsely participates in axonal transport of mitochondria}

a: Scheme of the experimental workflow; after transfection with a photoconvertible mitochondrial marker (mitoDendra) only the mitochondria of the cluster are converted from green to red by $455 \mathrm{~nm}$ laser illumination (boxed area, photoconversion indicated by yellow flash), while mitochondria in other localisations remained green.

Cells were subsequently imaged for $30 \mathrm{~min}$.

b: Primary cortical neurons (DIV7) were co-transfected with: NaV-emiRFP670 (top row), a live-cell-AISmarker linked to a near-infrared FP and mito-DENDRA, a mitochondrial targeted green-to-red-photoconvertible FP. middle shows green channel, bottom row red channel; before (left) and after (right) local photoconversion of the mitochondria in the cluster (enlarged in inserts and indicated by lightning bolt). Dashed line indicates the AIS, asterisks indicates the cell body.

c: Representative kymograph of the time-lapse imaging. Arrowheads show events of anterograde mitochondrial transport, cyan outline shows trafficking through and past the cluster, asterisk shows the stationary cluster in the proximal AIS.

d: Quantification of mitochondrial trafficking events in the AIS, split into mitochondria of the proximal cluster (red) and other mitochondria (green), shown as a fraction of each cells total trafficking events, Mean ${ }_{\text {Cluster- }}$ Mito $=0.146 ;$ Mean $_{\text {Other-Mito }}=0.853$; unpaired t-test: $* * *, p<0.0001,4$ biological replicates and $\mathrm{n}=6$.

\section{Fig. 4 Local mitochondrial impairment of the AIS-mitochondria-cluster leads to TAU-missorting}

a: MPN (DIV11) were incubated with the mitochondrial membrane potential marker TMRM (10nM) and the photoactivatable uncoupler Mito-PhotoDNP (MPD; 200nM) for 20min prior to photoactivation. MPD was only activated in "ROI A" by laser illumination, RGB-pseudocolouring of TMRM signal shows loss of mitochondrial membrane potential in the treated ROI A, but not in control ROI B.

b: Relative quantification of the mitochondrial membrane potential $(\Delta \Psi)$ from the experiment in (a), graph shows the decline and persisting low level of TMRM-fluorescence-intensity that is limited to the photoactivated ROI A; time-lapse-imaging for 7,5 min, measured every 6 s to reduce photobleaching.

c: Scheme of the experimental workflow to achieve local mitochondrial impairment in the AIS in live cultured neurons with MPD and measure the effects on somatodendritic TAU levels.

d: Representative live-imaging pictures of the AIS and mitochondria in DIV11 MPN, lightning bold shows the mitochondrial cluster in the proximal AIS where mitochondrial impairment was induced (boxed area). Asterisks indicate cell body.

e: Representative confocal images of cells after photoactivation, fixation and immunostaining for MAP2 and TAU. White dashed lines indicate AIS, rectangle shows section from $(\mathrm{d})$. Coloured dashed lines indicate the outline of the soma of the treated (green) and untreated cell (cyan). Magnifications show section of the soma (yellow boxes), note the slight elevation of TAU-fluorescence intensity in the soma of the cell that underwent photoactivation.

f-g: The experiment was repeated in 3 biological distinct replicates in both MPN and iPSC-derived-neurons, TAU-fluorescence intensity was measured in the soma of the treated cells (i.e. photoactivation of the mito-AIScluster). Untreated cells (i.e. also exposed to the MPD, but not photoactivated) in the same plate served as controls.

f: MPN, mean with SD, each point is one biological replicate; Control: Mean=1.0, $\mathrm{n}=5-16$.

Treated: Mean $=1.148, \mathrm{n}_{\text {Total }}=5$; unpaired-t-test, $* *, \mathrm{p}=0,0032$.

g: iPSC-derived neurons, mean with SD, each point is one biological replicate, Control: Mean=1.0, $\mathrm{n}=6-17$;

Treated: Mean $=1.119, \mathrm{n}_{\text {Total }}=4$; unpaired-t-test, *, $\mathrm{p}=0,0243$.

\section{Supplemental Figures:}


Suppl. 1 Respiratory chain inhibitors induce a global change of mitochondrial morphology Additional immunofluorescence staining of experiment from Fig. 1 for MAP2 and MTCO1 (mitochondrial marker.

a: Magnification shows healthy, elongated mitochondria forming a network.

b: Changed morphology of mitochondria after treatment with $50 \mu \mathrm{M}$ Rotenone for 2 hours, magnification shows scattered and rounded mitochondria as a sign of stress.

Suppl. 2 Somatodendritic activation of MPD does not result in TAU-missorting

a-b: The experiment from Fig. 4 e-g was also performed in co-transfected control cells in both MPN and iPSCderived-neurons, this time firing the UV-laser at the AIS-mitochondria cluster before the addition on MPD (a) or at a ROI of similar size in the somatodendritic compartment after addition of MPD.

TAU-fluorescence intensity was measured in the soma of the these cells, untreated cells in the same plate served

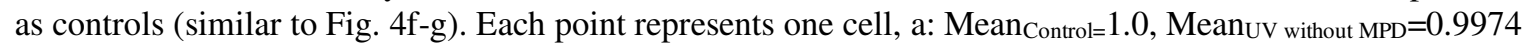
b: Mean Control $_{1}=1.0$, Mean Somato.act $=0.9966$.

\section{References}

[1] M. Robinson, B. Y. Lee, and F. T. Hane, "Recent Progress in Alzheimer's Disease Research, Part 2: Genetics and Epidemiology," J. Alzheimer's Dis., vol. 57, no. 2, pp. 317-330, Mar. 2017, doi: 10.3233/JAD-161149.

[2] H. Zempel and E.-M. Mandelkow, "Tau missorting and spastin-induced microtubule disruption in neurodegeneration: Alzheimer Disease and Hereditary Spastic Paraplegia," Mol. Neurodegener., vol. 10, no. 1, p. 68, Dec. 2015, doi: 10.1186/s13024-015-0064-1.

[3] M. Bell and H. Zempel, "SH-SY5Y-derived neurons: a human neuronal model system for investigating TAU sorting and neuronal subtype-specific TAU vulnerability," Rev. Neurosci., vol. 0, no. 0, Apr. 2021, doi: 10.1515/revneuro-2020-0152.

[4] H. Zempel and E. Mandelkow, "Mechanisms of Axonal Sorting of Tau and Influence of the Axon Initial Segment on Tau Cell Polarity," in Advances in Experimental Medicine and Biology, vol. 1184, Springer, 2019, pp. 69-77. doi: 10.1007/978-981-329358-8_6.

[5] H. Zempel et al., "Axodendritic sorting and pathological missorting of Tau are isoform-specific and determined by axon initial segment architecture," J. Biol. Chem., vol. 292, no. 29, pp. 12192-12207, Jul. 2017, doi: 10.1074/jbc.M117.784702.

[6] K. L. Hedstrom, Y. Ogawa, and M. N. Rasband, "AnkyrinG is required for maintenance of the axon initial segment and neuronal polarity," J. Cell Biol., vol. 183, no. 4, pp. 635-640, Nov. 2008, doi: 10.1083/jcb.200806112.

[7] C. Leterrier, "The Axon Initial Segment: An Updated Viewpoint," J. Neurosci., vol. 38, no. 9, pp. 2135-2145, Feb. 2018, doi: 10.1523/JNEUROSCI.1922-17.2018.

[8] S. L. Jones and T. M. Svitkina, "Axon Initial Segment Cytoskeleton: Architecture, Development, and Role in Neuron Polarity," Neural Plast., vol. 2016, pp. 1-19, 2016, doi: $10.1155 / 2016 / 6808293$.

[9] S. F. B. van Beuningen et al., "TRIM46 Controls Neuronal Polarity and Axon Specification by Driving the Formation of Parallel Microtubule Arrays," Neuron, vol. 88, no. 6, pp. 1208-1226, Dec. 2015, doi: 10.1016/j.neuron.2015.11.012.

[10] X. Li, Y. Kumar, H. Zempel, E.-M. Mandelkow, J. Biernat, and E. Mandelkow, "Novel diffusion barrier for axonal retention of Tau in neurons and its failure in neurodegeneration," EMBO J., vol. 30, no. 23, pp. 4825-4837, Nov. 2011, doi: 10.1038/emboj.2011.376.

[11] K. J. Kopeikina et al., "Tau accumulation causes mitochondrial distribution deficits in neurons in a mouse model of tauopathy and in human Alzheimer's disease brain," Am. J. Pathol., vol. 179, no. 4, pp. 2071-2082, Oct. 2011, doi: 
10.1016/j.ajpath.2011.07.004.

[12] R. H. Swerdlow, J. M. Burns, and S. M. Khan, "The Alzheimer's disease mitochondrial cascade hypothesis: Progress and perspectives," Biochim. Biophys. Acta - Mol. Basis Dis., vol. 1842, no. 8, pp. 1219-1231, Aug. 2014, doi: 10.1016/j.bbadis.2013.09.010.

[13] A. Mandal and C. M. Drerup, "Axonal Transport and Mitochondrial Function in Neurons," Frontiers in Cellular Neuroscience, vol. 13. Frontiers Media S.A., Aug. 09, 2019. doi: 10.3389/fncel.2019.00373.

[14] M. Manczak, M. J. Calkins, and P. H. Reddy, "Impaired mitochondrial dynamics and abnormal interaction of amyloid beta with mitochondrial protein Drp1 in neurons from patients with Alzheimer's disease: Implications for neuronal damage," Hum. Mol. Genet., vol. 20, no. 13, pp. 2495-2509, Jul. 2011, doi: 10.1093/hmg/ddr139.

[15] P. J. Flannery and E. Trushina, "Mitochondrial dynamics and transport in Alzheimer's disease," Mol. Cell. Neurosci., vol. 98, no. June, pp. 109-120, Jul. 2019, doi: 10.1016/j.mcn.2019.06.009.

[16] G. L. Rintoul and I. J. Reynolds, "Mitochondrial trafficking and morphology in neuronal injury," Biochimica et Biophysica Acta - Molecular Basis of Disease, vol. 1802, no. 1. Elsevier, pp. 143-150, Jan. 01, 2010. doi: 10.1016/j.bbadis.2009.09.005.

[17] D. Debanne, E. Campanac, A. Bialowas, E. Carlier, and G. Alcaraz, “Axon physiology," Physiol. Rev., vol. 91, no. 2, pp. 555-602, Apr. 2011, doi: 10.1152/physrev.00048.2009.

[18] R. S. Chaves et al., "Presence of insoluble Tau following rotenone exposure ameliorates basic pathways associated with neurodegeneration," IBRO Reports, vol. 1, pp. 32-45, Dec. 2016, doi: 10.1016/j.ibror.2016.09.001.

[19] H. Zempel and E. M. Mandelkow, "Tracking Tau in neurons: How to grow, fix, and stain primary neurons for the investigation of Tau in all developmental stages," in Methods in Molecular Biology, vol. 1523, Humana Press Inc., 2017, pp. 327-334. doi: 10.1007/978-1-4939-6598-4_20.

[20] C. Wang et al., "Scalable Production of iPSC-Derived Human Neurons to Identify TauLowering Compounds by High-Content Screening," Stem Cell Reports, vol. 9, no. 4, pp. 1221-1233, Oct. 2017, doi: 10.1016/j.stemcr.2017.08.019.

[21] S. Bachmann, J. Linde, M. Bell, M. Spehr, H. Zempel, and G. Zimmer-Bensch, "DNA methyltransferase 1 (Dnmt1) shapes neuronal activity of human ipsc-derived glutamatergic cortical neurons," Int. J. Mol. Sci., vol. 22, no. 4, pp. 1-14, Feb. 2021, doi: 10.3390/ijms22042034.

[22] Y. Miyaoka et al., "Isolation of single-base genome-edited human iPS cells without antibiotic selection," Nat. Methods, vol. 11, no. 3, pp. 291-293, Feb. 2014, doi: 10.1038/nmeth.2840.

[23] J. Schindelin et al., "Fiji: An open-source platform for biological-image analysis," Nature Methods, vol. 9, no. 7. Nature Publishing Group, pp. 676-682, Jul. 28, 2012. doi: 10.1038/nmeth.2019.

[24] A. S. Dumitrescu, M. D. Evans, and M. S. Grubb, "Evaluating Tools for Live Imaging of Structural Plasticity at the Axon Initial Segment," Front. Cell. Neurosci., vol. 10, no. NOV2016, Nov. 2016, doi: 10.3389/fncel.2016.00268.

[25] H. Zempel et al., "Amyloid- $\beta$ oligomers induce synaptic damage via Tau-dependent microtubule severing by TTLL6 and spastin," EMBO J., vol. 32, no. 22, pp. 29202937, 2013, doi: 10.1038/emboj.2013.207. 
[26] C. G. Dotti, C. A. Sullivan, and G. A. Banker, "The establishment of polarity by hippocampal neurons in culture," J. Neurosci., vol. 8, no. 4, pp. 1454-1468, 1988, doi: 10.1523/jneurosci.08-04-01454.1988.

[27] T. Misgeld and T. L. Schwarz, "Mitostasis in Neurons: Maintaining Mitochondria in an Extended Cellular Architecture," Neuron, vol. 96, no. 3, pp. 651-666, Nov. 2017, doi: 10.1016/j.neuron.2017.09.055.

[28] S. Chalmers et al., "Selective Uncoupling of Individual Mitochondria within a Cell Using a Mitochondria-Targeted Photoactivated Protonophore," J. Am. Chem. Soc., vol. 134, no. 2, pp. 758-761, Jan. 2012, doi: 10.1021/ja2077922.

[29] F. Höfflin et al., "Heterogeneity of the axon initial segment in interneurons and pyramidal cells of rodent visual cortex," Front. Cell. Neurosci., vol. 11, Nov. 2017, doi: 10.3389/fncel.2017.00332.

[30] W. Hu, C. Tian, T. Li, M. Yang, H. Hou, and Y. Shu, "Distinct contributions of Nav1.6 and Nav1.2 in action potential initiation and backpropagation," Nat. Neurosci., vol. 12, no. 8, pp. 996-1002, Aug. 2009, doi: 10.1038/nn.2359.

[31] A. H. Pham, J. M. Mccaffery, and D. C. Chan, "Mouse lines with photo-activatable mitochondria to study mitochondrial dynamics," Genesis, vol. 50, no. 11, pp. 833-843, Nov. 2012, doi: 10.1002/dvg.22050.

[32] R. N. Dimova and D. V. Markov, "Changes in the mitochondria in the initial part of the axon during regeneration," Acta Neuropathol., vol. 36, no. 3, pp. 235-242, 1976, doi: 10.1007/BF00685367.

[33] C. Nakada et al., "Accumulation of anchored proteins forms membrane diffusion barriers during neuronal polarization,” Nat. Cell Biol., vol. 5, no. 7, pp. 626-632, 2003, doi: $10.1038 /$ ncb1009.

[34] B. Winckler, P. Forscher, and I. Mellman, "A diffusion barrier maintains distribution of membrane proteins in polarized neurons," Nature, vol. 397, no. 6721, pp. 698-701, 1999, doi: 10.1038/17806.

[35] D. Pathak, K. J. Sepp, and P. J. Hollenbeck, "Evidence that myosin activity opposes microtubule-based axonal transport of mitochondria," J. Neurosci., vol. 30, no. 26, pp. 8984-8992, Jun. 2010, doi: 10.1523/JNEUROSCI.1621-10.2010.

[36] J.-S. Kang et al., "Docking of Axonal Mitochondria by Syntaphilin Controls Their Mobility and Affects Short-Term Facilitation," Cell, vol. 132, no. 1, pp. 137-148, Jan. 2008, doi: 10.1016/j.cell.2007.11.024.

[37] S. Aronov, G. Aranda, L. Behar, and I. Ginzburg, "Axonal tau mRNA localization coincides with tau protein in living neuronal cells and depends on axonal targeting signal," J. Neurosci., vol. 21, no. 17, pp. 6577-6587, Sep. 2001, doi: 10.1523/jneurosci.21-17-06577.2001.

[38] M. Tapia et al., "GSK3 and $\beta$-catenin determines functional expression of sodium channels at the axon initial segment," Cellular and Molecular Life Sciences, vol. 70, no. 1. Springer, pp. 105-120, Jan. 05, 2013. doi: 10.1007/s00018-012-1059-5.

[39] M. Llorens-Martín, J. Jurado, F. Hernández, and J. Ávila, “GSK-3ß, a pivotal kinase in Alzheimer disease," Frontiers in Molecular Neuroscience, vol. 7, no. MAY. Frontiers Research Foundation, p. 46, May 21, 2014. doi: 10.3389/fnmol.2014.00046.

[40] H. Hongo et al., "Glycogen synthase kinase-3 $\beta$ activation mediates rotenone-induced cytotoxicity with the involvement of microtubule destabilization," Biochem. Biophys. Res. Commun., vol. 426, no. 1, pp. 94-99, 2012, doi: 10.1016/j.bbrc.2012.08.042. 

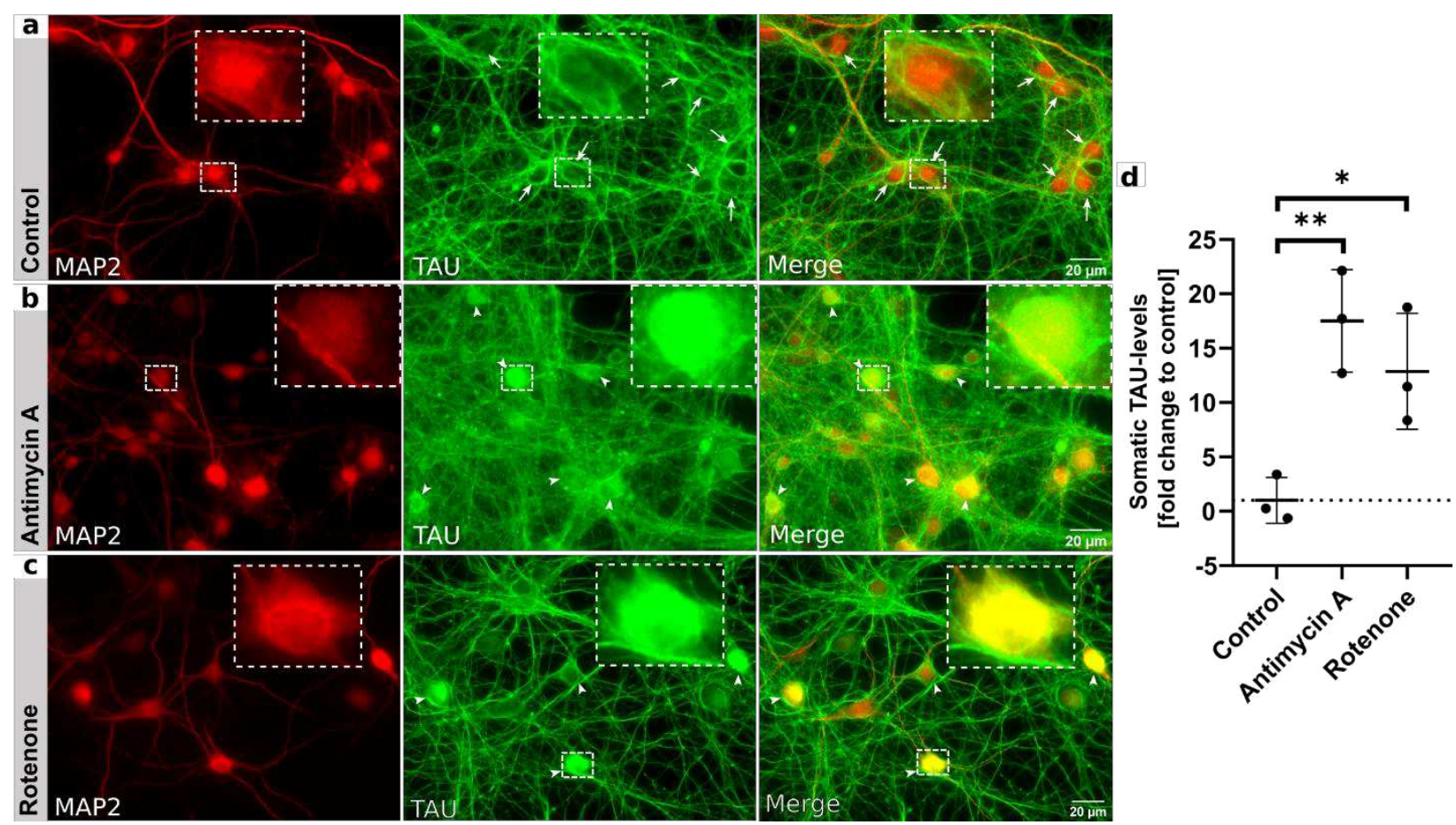

Fig. 1 


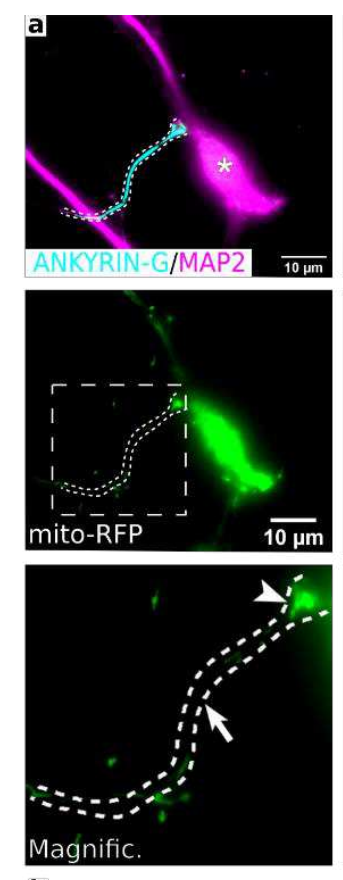

h

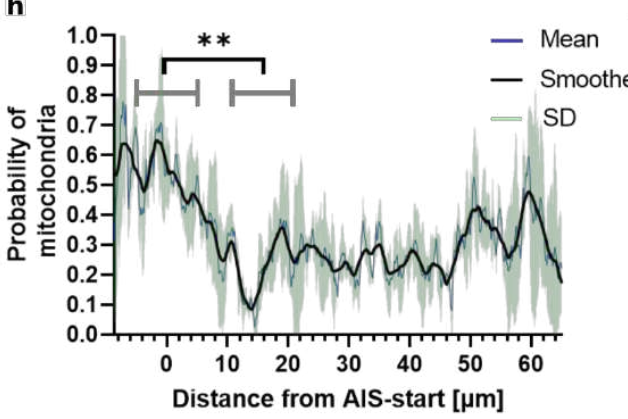

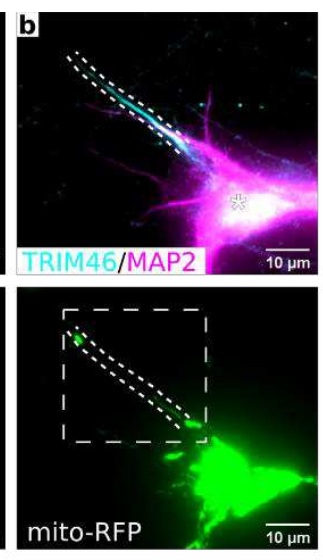

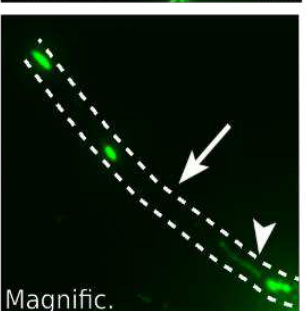

$\mathrm{mT}$

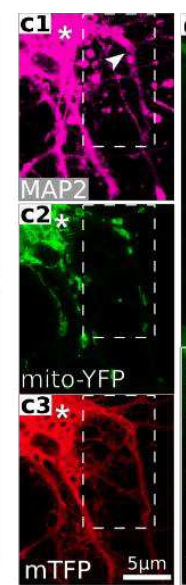

$g$ yly

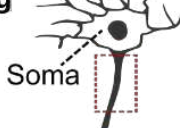

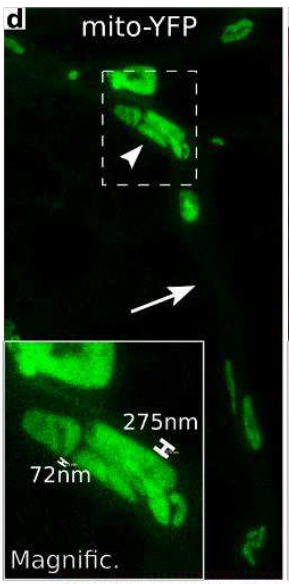

Axon

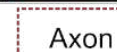

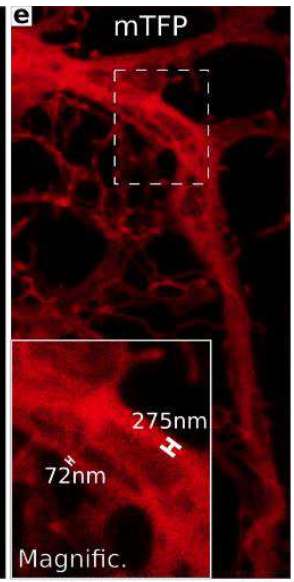

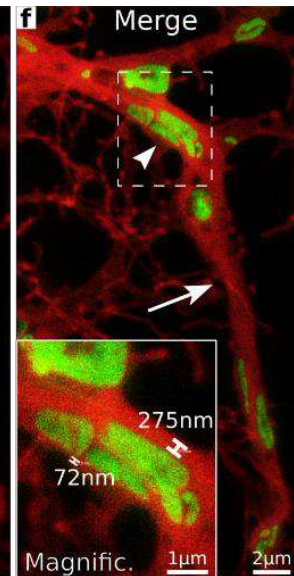

Axon Initial

Magnific. $\quad 1 \mu \mathrm{m} \quad 2 \mu$ Hillock Segment (AIS)

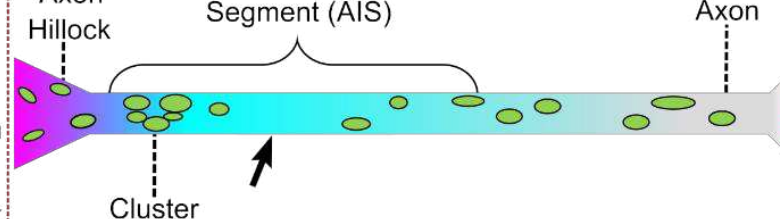

i
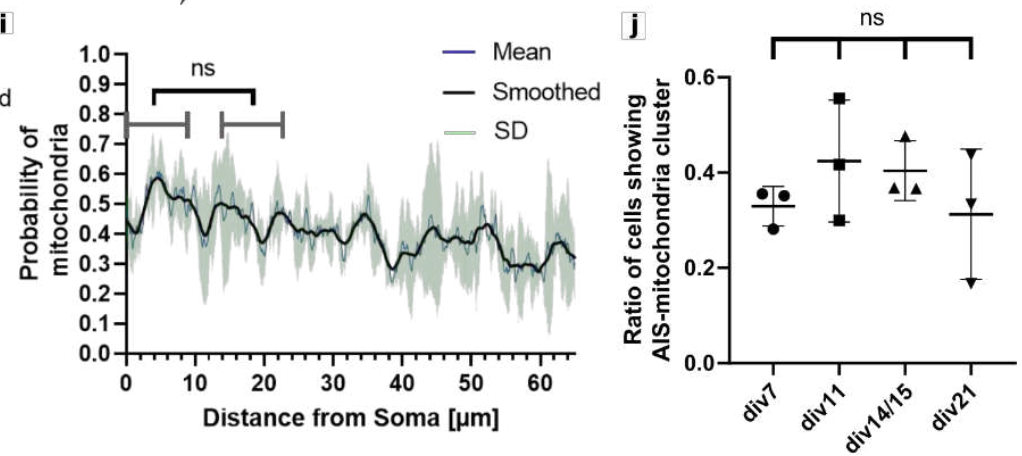

Fig. 2 

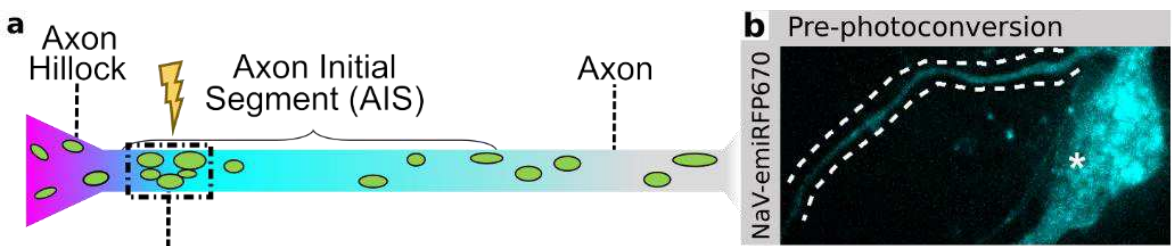

Post-photoconversion

Cluster

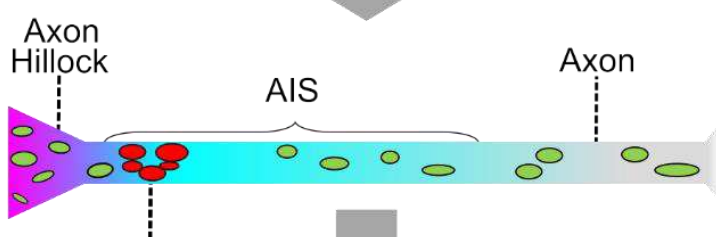

Cluster
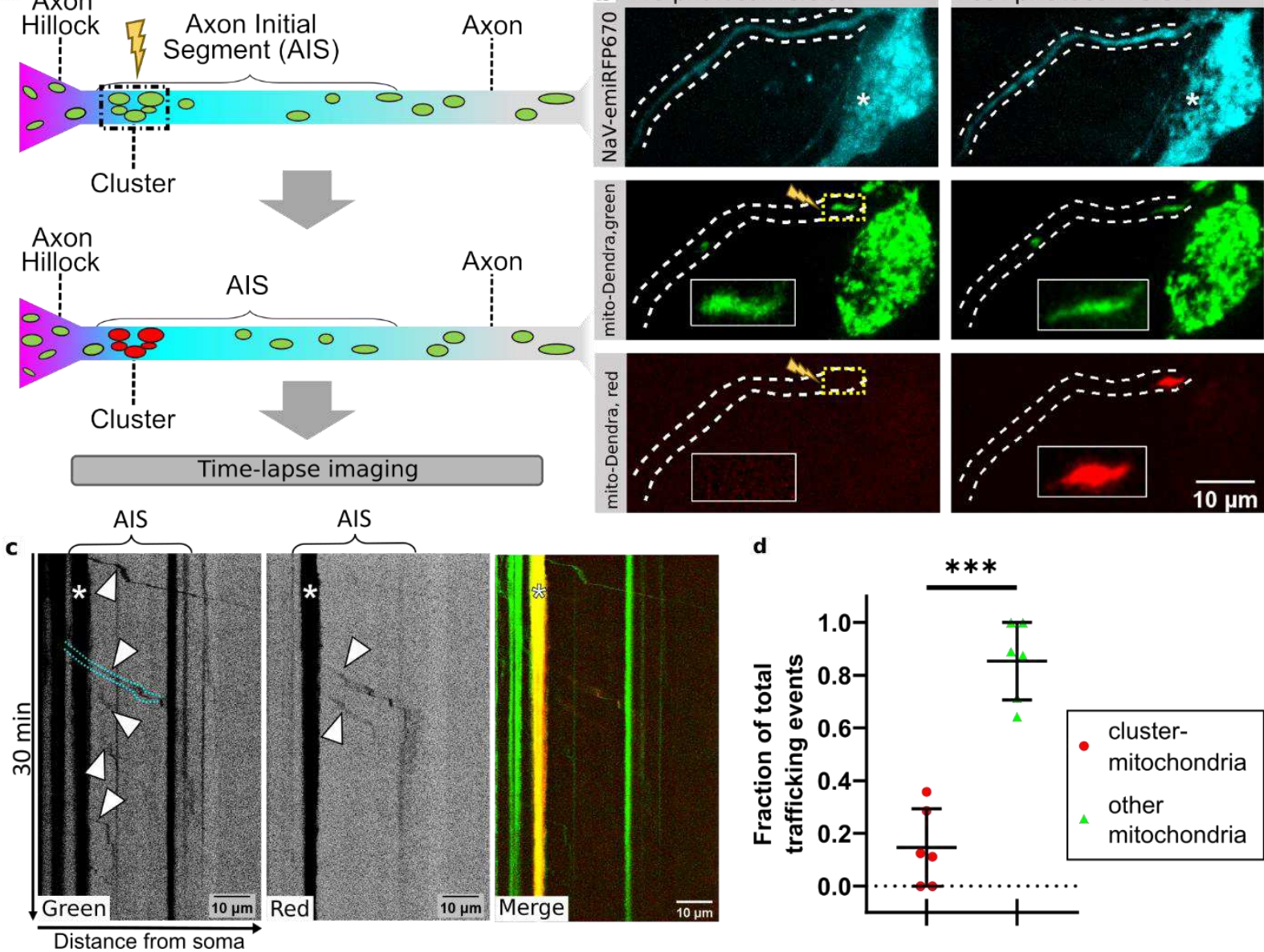

d
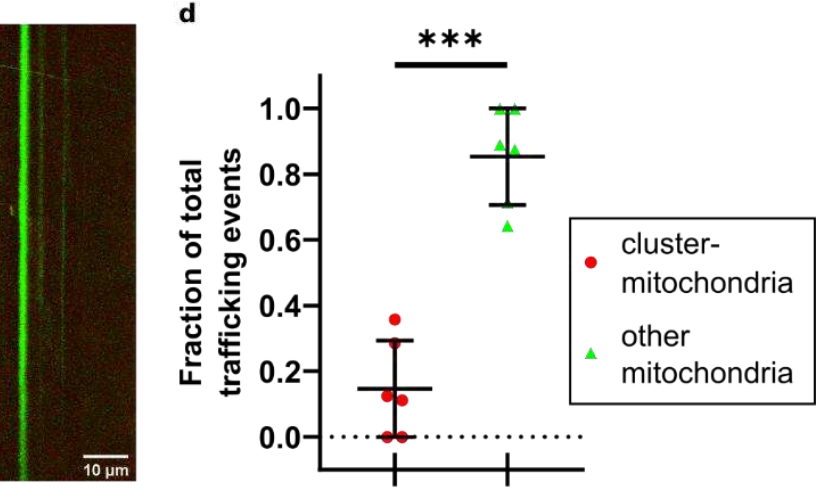

Fig. 3 

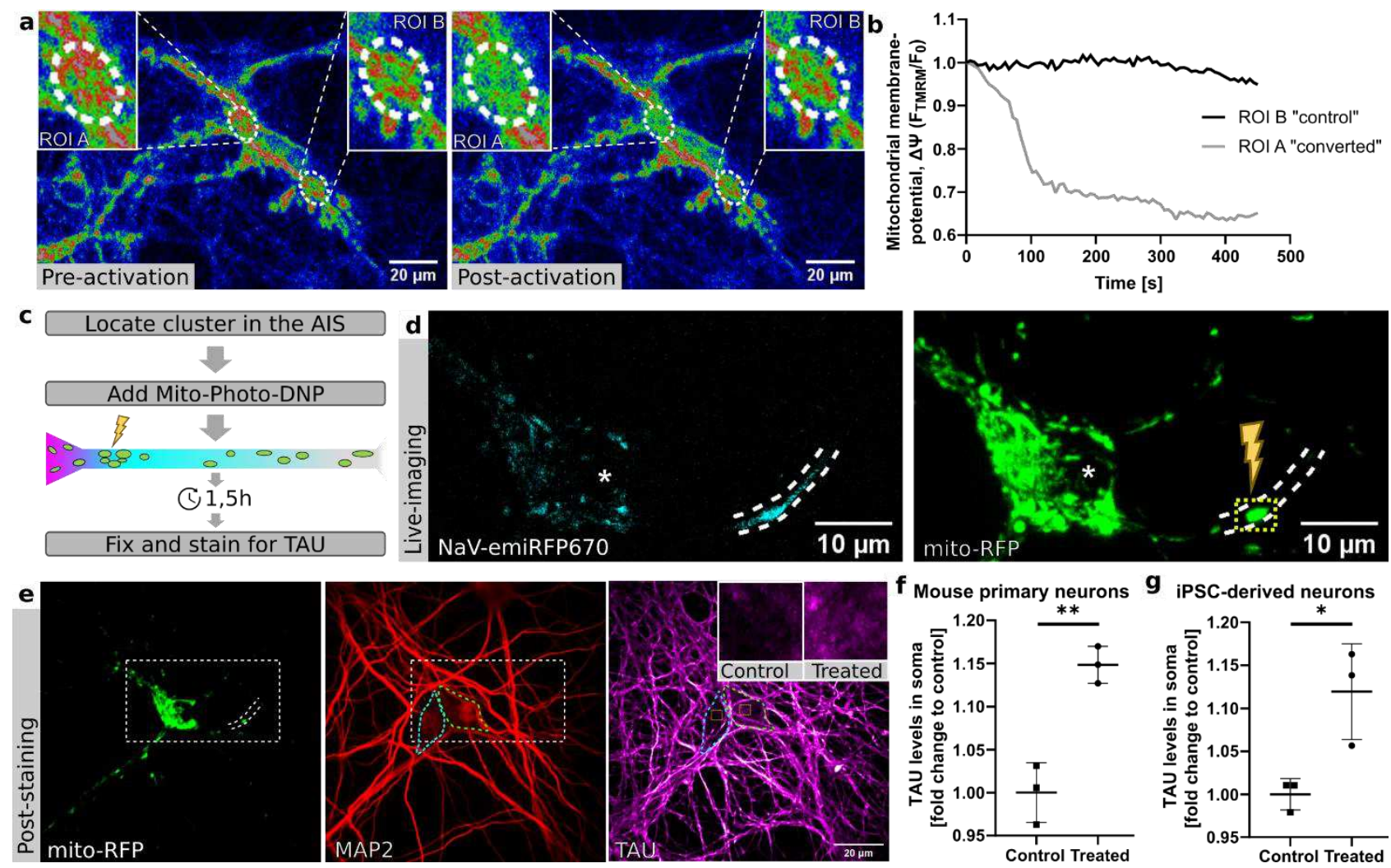

Fig. 4 


\section{Supplementary Files}

This is a list of supplementary files associated with this preprint. Click to download.

- SupplementalFiguresCaption.docx

- Suppl.FiguresNTHZ.pdf 